\title{
Pancreatic Vipoma
}

National Cancer Institute

\section{Source}

National Cancer Institute. Pancreatic Vipoma. NCI Thesaurus. Code C95599.

A usually malignant pancreatic neuroendocrine tumor producing vasoactive intestinal peptide (VIP). It is associated with watery diarrhea, hypokalemia, and hypochlorhydria or achlorhydria. One third of cases are metastatic at the time of diagnosis. 